\title{
Investigating the Relationship Between IGF-I, IGF-II, and IGFBP-3 Concentrations and Later-Life Cognition and Brain Volume
}

Antoine Salzmann, ${ }^{1}$ Sarah-Naomi James, ${ }^{1}$ Dylan M. Williams, ${ }^{1,2}$ Marcus Richards, ${ }^{1}$ Dorina Cadar, ${ }^{3}$ Jonathan M. Schott, ${ }^{4}$ William Coath, ${ }^{4}$ Carole H. Sudre, ${ }^{4,5}$ Nishi Chaturvedi, ${ }^{1}$ and Victoria Garfield ${ }^{1}$

${ }^{1}$ MRC Unit for Lifelong Health and Ageing at UCL, University College London, London, UK; ${ }^{2}$ Department
of Medical Epidemiology and Biostatistics, Karolinska Institutet, Stockholm, Sweden; ${ }^{3}$ Department
of Behavioural Science and Health, University College London, London, UK; ${ }^{4}$ Department of
Neurodegenerative Disease, The Dementia Research Centre, UCL Queen Square Institute of Neurology,
London, UK; and ${ }^{5}$ School of Biomedical Engineering and Imaging Sciences, King's College London,
London, UK

ORCiD numbers: 0000-0001-7877-9876 (A. Salzmann); 0000-0002-3800-4416 (S.-N. James); 0000-0001-9191-2192 (M. Richards); 0000-0003-1398-5841 (D. Cadar); 0000-0003-2059-024X (J. M. Schott); 0000-0002-3976-9461 (W. Coath); 0000-0002-6211-2775 (N. Chaturvedi); 0000-0002-5127-0890 (V. Garfield).

Received: 14 October 2020; Editorial Decision: 19 February 2021; First Published Online: 25 February 2021; Corrected and Typeset: 17 April 2021.

\begin{abstract}
Background: The insulin/insulin-like signaling (IIS) pathways, including insulin-like growth factors (IGFs), vary with age. However, their association with late-life cognition and neuroimaging parameters is not well characterized.

Methods: Using data from the British 1946 birth cohort, we investigated associations of IGF-I, IGF-II and IGF binding protein 3 (IGFBP-3; measured at 53 and 60-64 years of age) with cognitive performance [word-learning test (WLT) and visual letter search (VLS) at 60-64 years and 69 years of age] and cognitive state [Addenbrooke's Cognitive Exam III (ACE-III) at 69-71 years of age], and in a proportion, quantified neuroimaging measures [whole brain volume (WBV), white matter hyperintensity volume (WMHV), hippocampal volume (HV)]. Regression models included adjustments for demographic, lifestyle, and health factors.

Results: Higher IGF-I and IGF-II at 53 years of age was associated with higher ACE-III scores [ 0.07 95\% confidence interval $(\mathrm{Cl})(0.02,0.12)$; score $_{\mathrm{ACE}-\mathrm{III}} 89.48(88.86,90.1)$, respectively). IGF-II at 53 years of age was additionally associated with higher WLT scores [score WLT $_{20}(19.35,20.65)$ ]. IGFBP-3 at 60 to 64 years of age was associated with favorable VLS score at 60 to 64 and 69 years of age $[ß 0.07(0.01,0.12) ; ß 0.07(0.02,0.12)$,
\end{abstract}


respectively], higher memory and cognitive state at 69 years of age $[ß 0.07(0.01,0.12) ; \beta$ $0.07(0.01,0.13)$, respectively], and reduced WMHV $[ß-0.1(-0.21,-0.00)]$. IGF-I/IGFBP-3 at 60 to 64 years of was associated with lower VLS scores at 69 years of age [ $B-0.08$ $(-0.15,-0.02)]$.

Conclusions: Increased measure in IIS parameters (IGF-I, IGF-II, and IGFBP-3) relate to better cognitive state in later life. There were apparent associations with specific cognitive domains (IGF-II relating to memory; IGFBP-3 relating to memory, processing speed, and WMHV; and IGF-I/IGFBP-3 molar ratio related to slower processing speed). IGFs and IGFBP-3 are associated with favorable cognitive function outcomes.

Key Words: IGF-I, IGF-II, cognition, aging, epidemiology, brain volume

The growth hormone (GH)/insulin-like growth factor (IGF) signaling pathway is thought to play a key role in aging health and the determination of age-related diseases. In humans, this pathway is regulated through the somatotropic axis, which consists mainly of GH, IGF, and their respective binding proteins (1). IGF-I binds to IGF binding proteins (IGFBP) in high-affinity complexes to prevent degradation and binding to the IGF-I receptor (IGF1R), thus leaving concentrations of "free" circulating IGF-I relatively low. As such, the ratio of IGF-I to binding proteins provides an indication of bioavailability (1-3), while also mediating its effect via IGF1R; IGF-II bioavailability is also regulated by the IGF2 receptor (4). IGFBP-3, however, has also been shown to have IGF-independent associations on phenotypes (5).

Whereas research supports an important role of insulin/ insulin growth factor-like signaling (IIS) in early brain development and neuroplasticity, as evidenced by developmental defects associated with mutations in the IGF1R gene, its role in the maintenance of brain function is less well understood $(6,7)$. Observational studies are conflicting, with some reporting protective, detrimental, U-shaped and null associations $(8,9)$. The relationship between IGF-II and cognition and neurological health, on the other hand, remains largely uncharacterized, with only 1 study to date, which reported raised IGF-II concentrations in association with better cognition, while no association of IGF-I was observed (10)

The MRC National Survey for Health and Development (NSHD; also known as the 1946 British Birth Cohort), provides an ideal opportunity to study the relationship between measures of the IIS axis through mid-life and cognition and brain volume in later life (11). IGF-I and -II collection at multiple time points may help resolve previous discrepant findings by considering differential associations of age and duration of follow-up. Additionally the NSHD has a wealth of data across the life course, allowing us to control for potential confounders that have been omitted or based on recalled data in past studies, such as early-life cognition and affective symptoms. Finally, neuroimaging data enables associations with brain volume and neurovascular health to be studied in tandem with cognitive function.

We aimed to examine the independent associations of IGF-I, IGF-II, and IGFBP-3 measured at ages 53 and 60 to 64 with cognitive function measured at ages 60 to 64 and 69 and volumetric and cerebrovascular neuroimaging parameters [total brain, hippocampal hyperintensity volume, and white matter hyperintensity volumes (WMHV)] at age 69 to 71 .

\section{Methods}

\section{Study participants}

The NSHD recruited 5362 participants born within 1 week in March 1946 in England, Scotland, and Wales. The cohort has remained in continuous follow-up from birth to date, with 24 sweeps of investigation (12). Cognitive function at age 69 was our primary outcome. At this age, 2149 participants were interviewed. The loss to follow-up was due to death (19.1\%), refusal $(20.6 \%)$, emigration or being temporarily abroad $(10.8 \%)$, or because the participant could not be contacted or traced $(7.8 \%)$. Figure 1 outlines the number of participants with information concerning exposure and outcome variables and the final sample $(\mathrm{n}=1762)$ used for analysis.

We additionally examined associations with neuroimaging outcomes in a subsample who had participated in the neuroimaging substudy, Insight 46 (13). Details have previously been published (13), but in brief this study included 502 individuals who underwent positron emission tomography-magnetic resonance imaging (PET-MRI) scanning using a single Biograph mMR 3T PET-MRI scanner (Siemens Healthcare, Erlangen, Germany), with simultaneous acquisition of PET/MRI data, including volumetric (1.1 mm isotropic) T1 and fluid-attenuated inversion recovery (FLAIR) sequences (13). Volumetric T1-weighted and FLAIR images underwent visual quality control 
prior to processing following an automated pipeline (13). Relevant imaging measures were isolated as follows: total brain volume using multi-atlas propagation and segmentation (14), mean hippocampal volume using similarity and truth estimation for propagated segmentations (15), total intracranial volume using SPM12 (Wellcome Centre for Human Neuroimaging) and (WMHV) using Bayesian model selection $(16,17)$. WMHV was isolated from 3D T1 and FLAIR images followed by visual inspection to generate a global WMHV, including subcortical grey matter but excluding infratentorial regions $(17,18)$. Eligibility criteria, the study protocol, and a comparison of those who did and who did not participate in Insight 46 have been described in detail elsewhere $(13,18)$. Of the 502 Insight 46 participants, complete neuroimaging and Addenbrooke's Cognitive Exam-III (ACE-III) data were available for 378 .

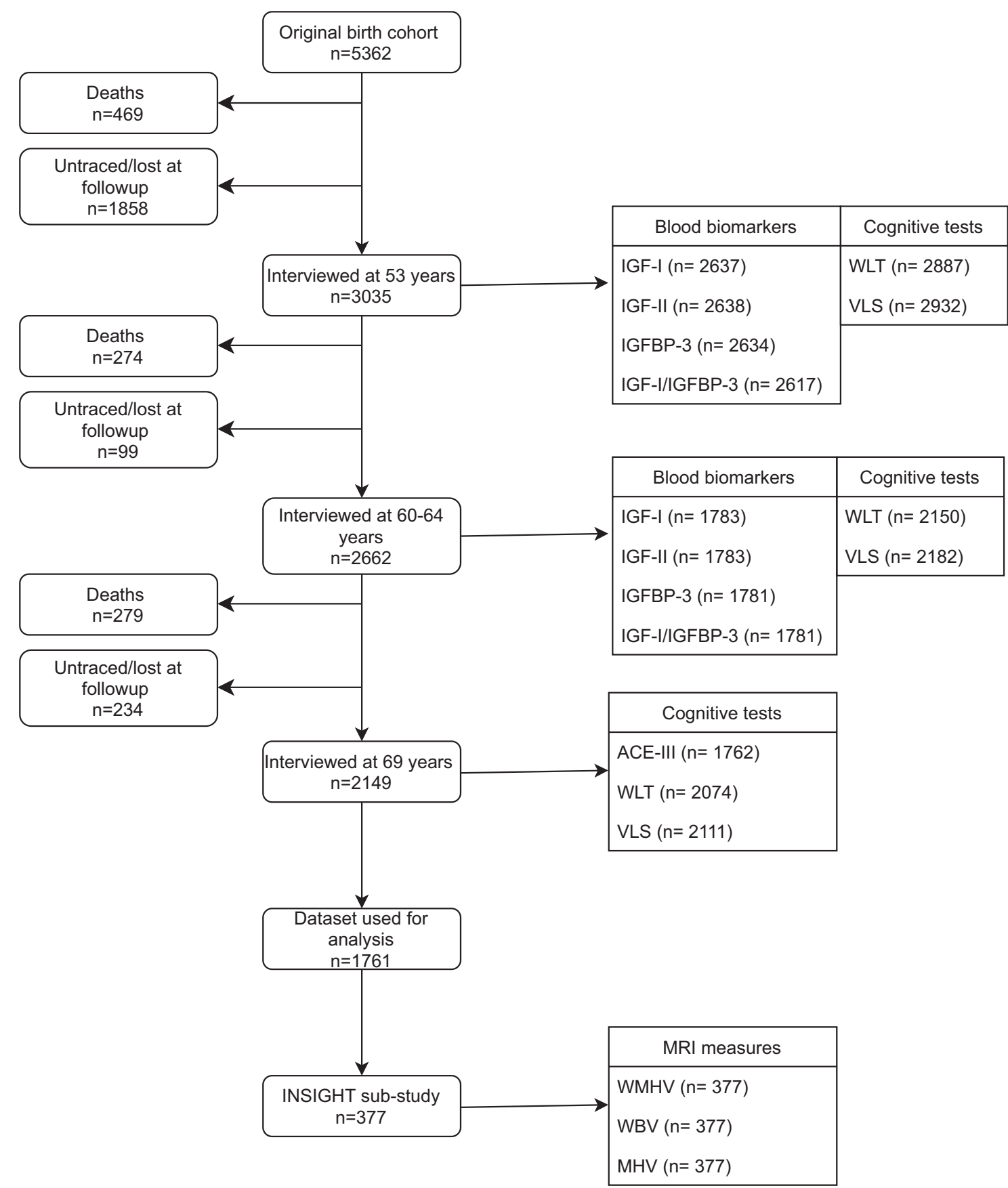

Figure 1. NSHD study flow diagram. Abbreviations: ACE-III, Addenbrookes Cognitive Exam III; IGF-I, insulin growth factor I; IGF-II, insulin growth factor II; IGFBP-3, insulin growth factor binding protein 3; MHV, mean hippocampal volume; VLS, visual letter search test; WBV, whole brain volume; WLT, word learning test; WMHV, white matter hyperintensity volume. 


\section{Measures}

\section{IGF-I, IGF-II, and IGFBP-3 concentrations}

Blood samples were collected at age 53 (nonfasting), and age 60 to 64 (fasting). Samples were stored at $-80^{\circ} \mathrm{C}$ and assayed together. IGF-I, IGF-II, and IGFBP-3 concentrations were obtained by radioimmunoassay using standard protocols in the same laboratory, as previously described (19). The intra-assay coefficients of variation for IGF-I, IGF-II, and IGFBP-3 were $3.4 \%, 2.8 \%$, and $3.9 \%$, respectively. The interassay coefficients of variation were $13.7 \%$, $7.4 \%$, and $11.7 \%$ for IGF-I, IGF-II, and IGFBP-3, respectively (20). IGF-I, IGF-II, and IGFBP-3 values were converted from $\mathrm{ng} / \mathrm{mL}$ to standard SI units: nmol/L (21). IGF-I/ IGFBP-3 values were calculated as molar ratios as an indicator of IGF-I bioavailability. Repeat measures ranged between 1117 and 1127 participants for IGF-I/IGFBP-3 ratio and IGFBP-3, respectively.

\section{Cognitive performance measures}

Three separate tests were used to measure cognitive domains. At ages 60 to 64 and 69, standardized versions of a word-learning test (WLT) and a visual letter search test (VLS) were used to assess memory and processing speed, respectively. The WLT consists of a 15 -word learning task devised for the NSHD. Each word was shown to the participant for 2 seconds, and participants were asked to immediately write down as many words as they could remember. The task was repeated 3 times, and the scores were summed $(\max =45)$, which is representative of short-term episodic memory $(22,23)$. The VLS required participants to scan for the letters "P" and "W" among other letters and cross out as many as they could within 1 minute. Search speed score was based on how far the participant got in the grid search, with a maximum score of 600 signifying they had completed the whole grid (22).

The ACE-III is a test for cognitive state administered at age $69(24,25)$. The test assesses 5 domains, each with different score ranges: attention (0-18), memory (0-26), fluency (0-14), language (0-26), and visuospatial (0-16). The test was administered either online using an iPad (http:// www.acemobile.org/) or by paper if the former was not possible. Paper tests were scored by trained personnel $(24,26)$. Unlike most tests of cognitive state, the ACE-III total score $(\max =100)$ has a quasi-normal distribution, making it appropriate for parametric statistical analyses.

\section{Covariates}

Covariates were selected a priori, based on previous studies and added into our models successively. Model 1 had sex,
Model 2 included additional adjustments for childhood cognition and educational attainment, Model 3 added smoking status at the time of exposure, and Model 4 added affective symptoms at the time of outcome $(10,27,28)$. The same models were used when investigating the relationship between exposures and brain volume with total intracranial volume additionally controlled for in all volumetric outcome models.

Childhood cognition at age 8 was assessed by 4 tests administered by trained observers: reading comprehension, word pronunciation, vocabulary, and nonverbal reasoning. Total scores were standardized to the study population. Lifetime education attainment was based on the highest educational qualification by the age of 43 years (26) and categorized as (i) no qualification or vocational qualifications; (ii) ordinary, advanced secondary; and (iii) tertiary qualifications. Smoking status was obtained from questionnaires and classified into current smoker, ex-smoker, and never smoker at age 53 and 60 to 64 (29). Affective symptoms were self-reported at ages 60 to 64 and 69 years using the 28-item General Health Questionnaire (GHQ-28) (30). A threshold, set at the recommended 4 (noncase) $/ 5$ (case) cut, was imposed on the General Health Questionnaire total score to determine case-level symptoms $(30,31)$.

\section{Statistical analyses}

WMHV was $\log _{\mathrm{e}}$-transformed to account for skewness. Analysis of variance tests were used to examine exposure and outcome variable differences at different timepoints. Within the selected sample, missing data ranged from $0.1 \%$ to $27.9 \%$ for affective case symptoms (age 69) and IGF-I/IGFBP-3 molar ratio (age 60-64), respectively. To account for attrition at follow-up, missing data for exposure, WLT, VLS, and covariate variables were imputed using multiple imputation by chained equations. Imputation models included all model variables, except for volumetric outcomes. Datasets were imputed 20 times using a Bayesian linear regression model (32).

Linear regression models were used to assess the relationship between exposure and outcomes. Results are presented as standardized beta values with $95 \%$ confidence intervals $(\mathrm{CI})$. To determine whether the associations of IGFs and IGFBP-3 with cognition and brain volume differed by sex, which may warrant stratification, an interaction term for sex was tested at the $10 \%$ significance level for all exposures. No interaction was found between sex and exposure variables, so main models were not stratified by sex. In addition, a quadratic term for the exposure was tested to explore the possibility of nonlinear associations. A quadratic relationship was observed between IGF-II and general cognitive state and visual memory. Therefore, for associations with cognitive 
outcomes, IGF-II measurements were categorized into tertiles.

All statistical analyses were performed in $\mathrm{R}$ version 3.5.2 (33). Multiple imputation was performed using the "mice" package in R (32). Relevant R script is available upon request.

\section{Results}

\section{Sample characteristics}

Of the 1762 NSHD participants with cognitive state measured at age 69, $849(48 \%)$ were male and 913 (52\%) were female. The Insight 46 imaging substudy at age 69 to 71 (mean age: 70.67 years, SD: 0.67 ) included 378 of these 1762 NSHD participants. Of these, 194 (51\%) were male and $184(49 \%)$ female (Table 1). While those who attended the clinic at ages 53 and 60 to 64 were overall healthier-less likely to smoke, higher socioeconomic status, and were more educated-the concentration of IGF pathway components was similar between participants in the full sample and those included in the final analysis (data not shown).

In the entire cohort at age 60 to 64 , the overall pattern shows that IGF-I concentrations were lower, and IGFBP3 levels substantially lower than at age 53 (Table 1). As a result, IGF-I/IGFBP3 ratio was higher at age 60 to 64 than at age 53. IGF-II concentrations were lower at age 60 to 64 than at age 53. Visual memory (WLT) and processing speed (VLS) were lower with increasing age at investigation, as previously described (Table 1) (34).

\section{IGF-I}

Higher IGF-I concentrations at age 53 and 60 to 64 were associated with better cognitive function for the WLT and ACEIII cognitive measures, but not for the VLS (Fig. 2). These associations reached conventional levels of statistical significance for IGF-I at age 53 and ACE-III at age 69 ( $(0.07,95 \%$

Table 1. Variable information for the sample $[n=1762$ (NSHD) and $n=378$ (INSIGHT)] prior to imputation

\begin{tabular}{|c|c|c|c|c|c|}
\hline & \multicolumn{3}{|c|}{ NSHD (n = 1762) } & \multirow{2}{*}{$\frac{\text { Insight } 46(\mathrm{n}=378)}{\text { Aged } 69-71}$} & \multirow[b]{2}{*}{$P$-value ${ }^{a}$} \\
\hline & Aged 53 years & Aged $60-64$ years & Aged 69 years & & \\
\hline \multicolumn{6}{|l|}{ Exposures } \\
\hline IGF-I levels (nmol/L) & $26.22(9.00)$ & $23.00(7.71)$ & & & $<0.001$ \\
\hline IGFBP-3 levels (nmol/L) & $173.22(39.31)$ & $120.11(30.16)$ & & & $<0.001$ \\
\hline IGF-I/IGFBP-3 molar ratio & $0.16(0.06)$ & $0.20(0.06)$ & & & $<0.001$ \\
\hline IGF-II levels (nmol/L) & $99.82(32.36)$ & $87.86(38.60)$ & & & $<0.001$ \\
\hline \multicolumn{6}{|l|}{ Cognitive tests } \\
\hline Addenbrooke's Cognitive Examination score & & & $91.52(6.01)$ & & \\
\hline Visual letter search score & $283.52(73.34)$ & $268.25(70.54)$ & $263.56(74.32)$ & & $<0.001$ \\
\hline Word learning test score & $24.87(6.12)$ & $24.68(6.06)$ & $22.38(6.13)$ & & $<0.001$ \\
\hline \multicolumn{6}{|l|}{ Insight $46 \mathrm{MRI}$ measures } \\
\hline Age at scan & & & & $70.67(0.67)$ & \\
\hline Hippocampal volume (ml) & & & & $3.13(0.33)$ & \\
\hline Total intracranial volume $(\mathrm{ml})$ & & & & $1432.86(127.58)$ & \\
\hline White matter hyperintensity volume $\mathrm{e}^{b}(\mathrm{ml})$ & & & & $3.08(5.19)$ & \\
\hline Whole brain volume (ml) & & & & $1101.38(95.34)$ & \\
\hline \multicolumn{6}{|l|}{ Model covariates } \\
\hline Early life cognition & $0.14(0.69)$ & & & & \\
\hline \multicolumn{6}{|l|}{ Affective symptoms case status, $\mathrm{n}(\%)$} \\
\hline Noncase & $1313(81)$ & $1247(83)$ & $1505(86)$ & & \\
\hline Case & $316(19)$ & $264(17)$ & $251(14)$ & & \\
\hline \multicolumn{6}{|l|}{ Educational attainment, n (\%) } \\
\hline No qualifications/vocational only & $637(36)$ & & & & \\
\hline O-level/A-level or equivalent & $835(48)$ & & & & \\
\hline Higher & $272(16)$ & & & & \\
\hline \multicolumn{6}{|l|}{ Smoking status, n (\%) } \\
\hline Current smoker & $315(19)$ & $148(10)$ & $525(9)$ & & \\
\hline Ex-smoker & $842(51)$ & $875(58)$ & $1061(61)$ & & \\
\hline Never smoker & $507(30)$ & $489(32)$ & $525(30)$ & & \\
\hline
\end{tabular}

${ }^{a} P$-values were calculated using a 1 -way analysis of variance for continuous variables.

${ }^{b}$ Median and interquartile range presented for nonnormally distributed variables. 


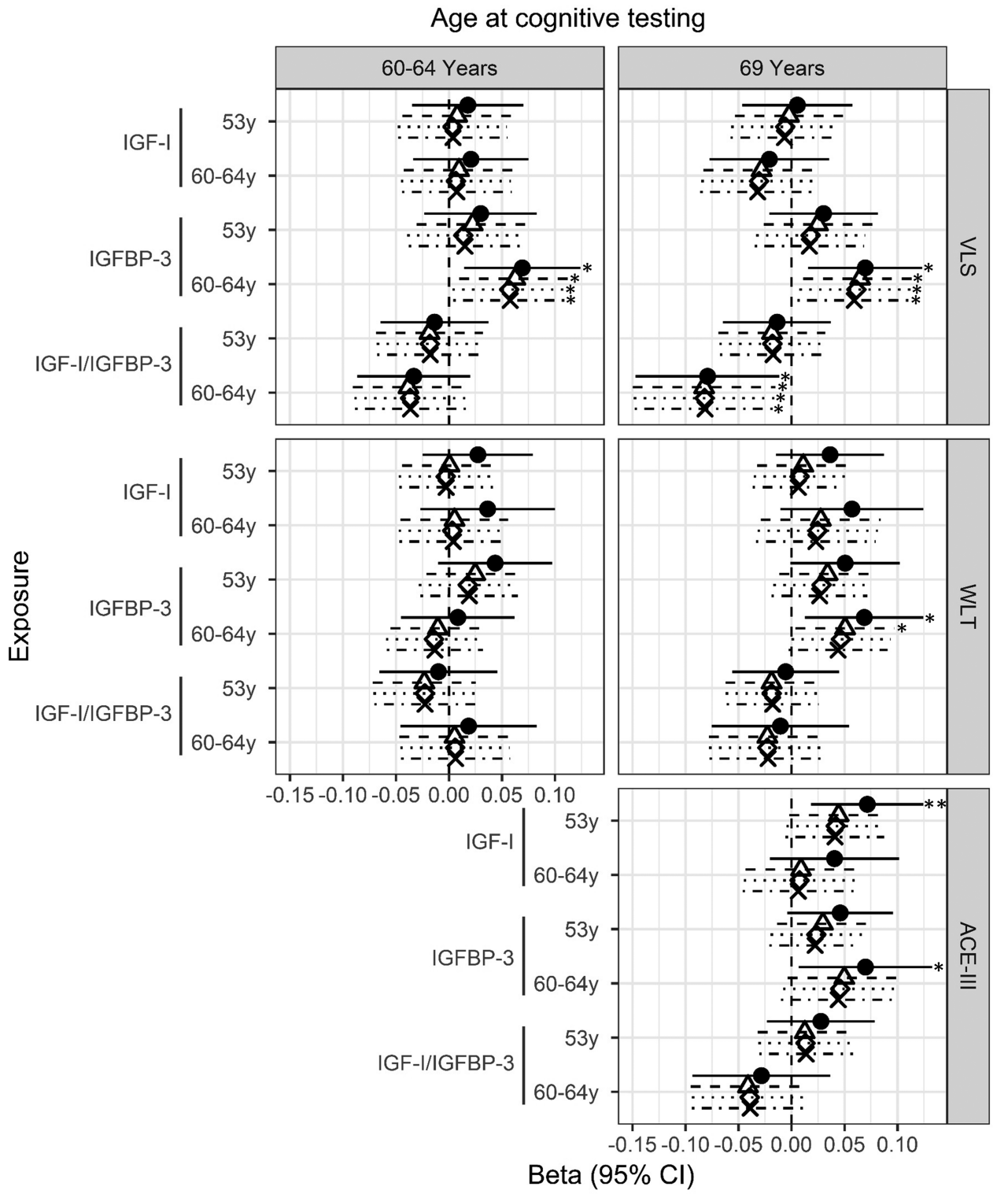

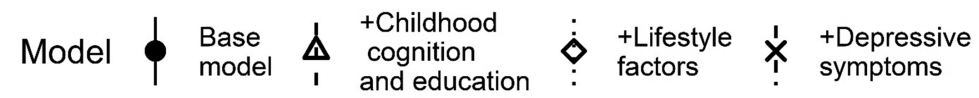

Figure 2. Beta coefficient and $95 \%$ confidence interval for the effect of IGF-I, IGFBP-3, and IGF-I/IGFBP-3 at ages 53 years and 60 to 64 years on visual memory (WLT) and processing speed (VLS) at ages 60 to 64 years and 69 years and cognitive state (ACE-III) at age 69 years. Models were adjusted for sex (base model); sex, early life cognition, and lifetime education (+childhood cognition and education model); sex, early life cognition, lifetime education, and smoking (+lifestyle factors); and sex, early life cognition, lifetime education, smoking, and depressive symptoms (+depressive symptoms). * significant at $P<0.05$; ${ }^{*}$ significant at $P<0.01$. Abbreviations: ACE-III, Addenbrookes Cognitive Exam III; IGF-I, insulin growth factor I; IGFBP3 , insulin growth factor binding protein $3 ; \mathrm{VLS}$, visual letter search test; WLT, word learning test.

CI $0.02,0.12, P=0.008)$. Adjustment for childhood cognition and educational attainment attenuated this estimate to B 0.04, 95\% CI 0.00, $0.09, P=0.063$. Further adjustment for smoking status and emotional symptoms did not alter the estimate (Fig. 2). Excluding participants with case-level affective symptoms at baseline had no qualitative impact on the magnitude or direction of associations (data not shown).

In the subjects with imaging available, higher concentrations of IGF-I at age 60 to 64 were directionally associated with a $8 \%$ lower WMHV at age 71 years $(95 \%$ CI $-19 \%$, $3 \%, P=0.142$ ), yet did not reach conventional levels of significance. Adjustment for the covariates did not affect this trend (Fig. 3).

\section{IGF-I/IGFBP-3 ratio}

IGF-I/IGFBP-3 ratio was associated with lower VLS and ACE-III scores (Fig. 2). Elevated IGF-I/IGFBP-3 

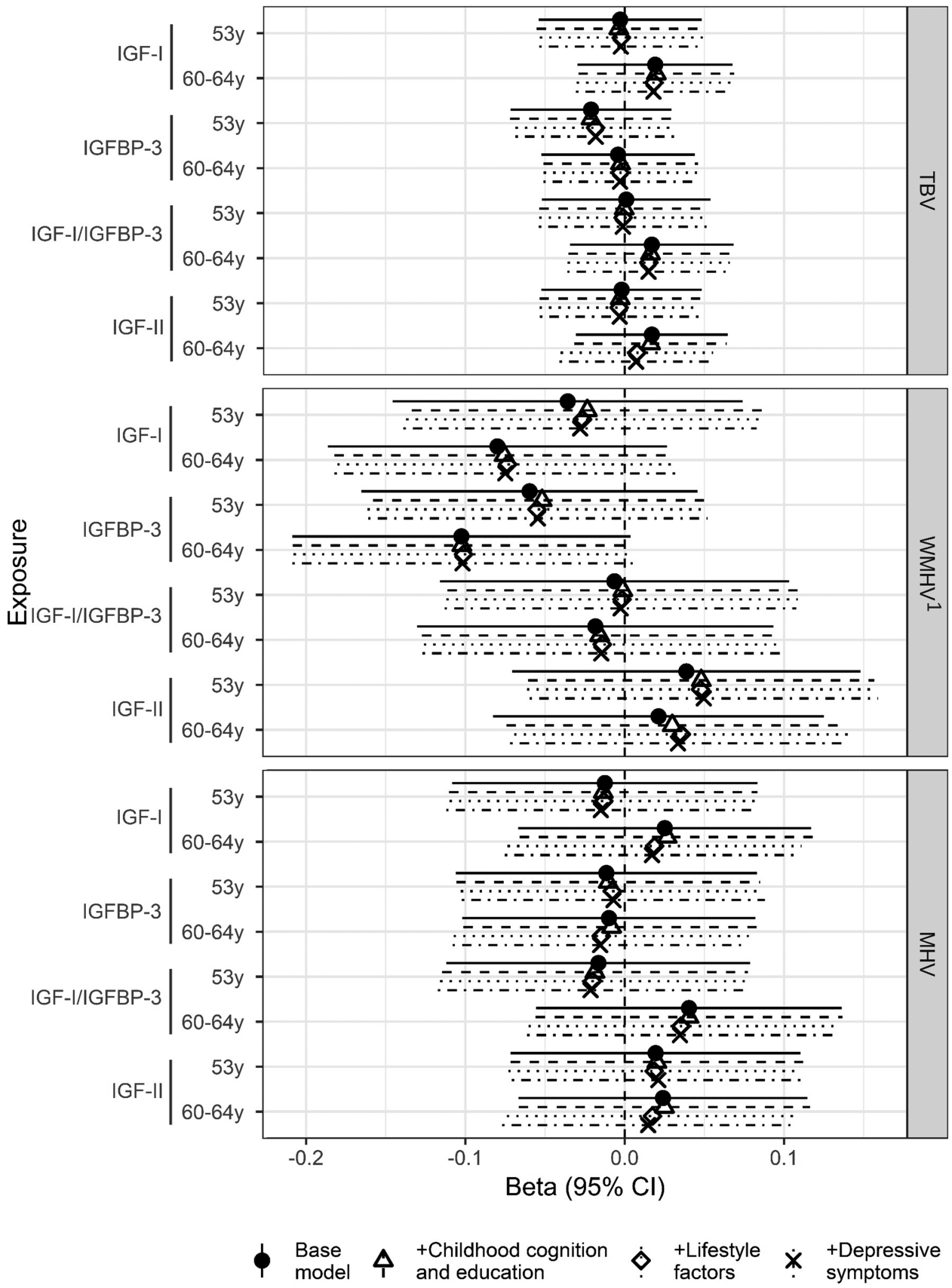

Figure 3. Beta coefficient and 95\% confidence interval for the effect of IGF-I, IGFBP-3, and IGF-I/IGFBP-3 at ages 53 years and 60 to 64 years on TBV, WMHV, and MHV at age 69 to 71 years in the Insight 46 substudy. Models were adjusted for sex and age at scan (base model); sex, early life cognition, and lifetime education (+childhood cognition and education model); sex, age at scan, early life cognition, lifetime education, and smoking (+lifestyle factors); and sex, age at scan, early life cognition, lifetime education, smoking, and depressive symptoms (+depressive symptoms). * significant at $P<0.05,{ }^{* *}$ significant at $P<0.01$. Abbreviations: MHV, mean hippocampal volume; TBV, total brain volume; WMHV, white matter hyperintensity volume. ${ }^{1}$ Effect sizes are shown on a logarithmic scale.

concentrations at age 60 to 64 were associated with lower VLS score at age $69(B-0.08,95 \%$ CI $-0.15,-0.02$, $P=0.019$ ) (Fig. 2). In contrast to IGF-I models, adjustment for childhood cognition and education did not attenuate effect sizes. No clear associations with brain volume were observed (Fig. 3).

\section{IGFBP-3}

Higher concentrations of IGFBP-3 at age 60 to 64 were associated with faster processing speed at age 60 to 64 and 69 ( $B 0.07,95 \%$ CI $0.01,0.12, P=0.014$ and $B 0.07,95 \%$ CI $0.02,0.12, P=0.012$, respectively) (Fig. 2). The addition of covariates did not affect this association (Fig. 2). Higher 
concentrations of IGFBP-3 at age 60 to 64 were additionally associated with higher scores for memory and cognitive state at age $69(B 0.07,95 \%$ CI $0.01,0.12, P=0.017$ and ß $0.07,95 \%$ CI $0.01,0.13, P=0.033$, respectively) (Fig. 2). Adjustment for education and childhood cognition attenuated these estimates to $B 0.05,95 \%$ CI $0: 00,0.10, P=0.034$ and $B 0.05,95 \%$ CI 0:00, $0.10, P=0.07$, respectively (Fig. 2 ).

Higher concentrations of IGFBP-3 at age 60 to 64 were additionally associated with a $10 \%$ lower WMHV at age 71 years ( $B-0.1,95 \% \mathrm{CI}-0.21,0.00, P=0.059)$ (Fig. 3). Adjustment for the covariates did not affect this association (Fig. 3).

\section{IGF-II}

Similarly to IGF-I, higher concentrations of IGF-II were associated with better cognitive function for the WLT and ACE-III cognitive measures, but not with VLS (Fig. 4). At age 53, the lowest tertile of circulating IGF-II concentration was associated with a lower ACE-III score at age 69 (score $_{\text {ACE-III }} 89.48,95 \%$ CI 88.86, 90.1, $P=0.022$, tertile 1) (Fig. 4). Similarly, the lowest tertile of IGF-II at age 53 was associated with lower memory at age 69 (score $_{\text {WLT }} 20$, $95 \%$ CI $19.35,20.65, P=0.085$, tertile 1 ). As for IGF-I/ IGFBP-3 ratio, no associations with brain volume were observed (Fig. 3).

\section{Discussion}

In this UK population sample, IGF-I and IGF-II concentrations at age 53 and IGF-I concentrations at age 60 to 64 , were positively associated with cognitive state at age 69 , assessed by the ACE-III and verbal memory but not by processing speed. Higher IGFBP-3 concentrations at age 60 to 64 were associated with overall higher cognition. Adjustment for childhood cognition and educational attainment attenuated these associations but did not wholly account for them. In addition, IGF-I and IGFBP-3 at age 60 to 64 were associated with lower white matter hyperintensity burden at age 69 to 71 . Multivariable adjustment did not notably alter these associations. Surprisingly, there was a trend toward worse cognitive state and processing speed in those with higher IGF-I/ IGFBP-3 ratio levels.

The positive relationship between IGF-I and general cognitive state reflects findings in the Nurses Health Cohort, which found an association between IGF-I and verbal memory attenuated after adjustment for educational attainment (35). Nurses Health Cohort participants were of similar age to NSHD participants at our second baseline (60-64 years) and the main follow-up point (69 years). Additionally, we suggest associations are similar in men and women and show that cognitive state is related to IGF-I sampled earlier in life, at age 53. Our findings conflict with the observation of an adverse (36) or U-shaped relation (37) of IGF-I on cognition. The former analyses were performed cross-sectionally in much older participants ( $\geq 95$ years), while the latter spanned ages 48 to 88 at follow-up. Age-dependent pleiotropic effects of IGF-I could potentially account these conflicting findings; specifically, IGF-I may act beneficially in middle age and early older age but has adverse effects in late older age. A U-shaped association may therefore be observed if participants span early and late older age.

The IGF pathway plays an essential role in brain development and homeostasis, including neurogenesis, neuronal differentiation, and angiogenesis $(8,38)$. Thus, the apparent beneficial association of increased pathway activation with cognitive and brain outcomes may be driven by a combination of developmental-with better cerebral development during fetal and early life and neuroprotective pathways-with increased neurogenesis and improved outcomes following cerebrovascular events (8,39-41). Prospective analyses of the Framingham Heart Study found that higher concentrations of IGF-I were associated with larger brain volumes in healthy participants, potentially suggestive of some protection against neurodegeneration (42). Association magnitudes were doubled in the parent $v s$ the offspring cohort (mean ages 79 years and 61 years, respectively), highlighting an important age dependency. While we did not observe an association between IGF-I and total brain volume, IGF-I was associated with lower WMHV, an indicator of cerebral small vessel damage. To our knowledge, no previous study has explored the association between IGF-I and cerebral small vessel disease. A 10-year prospective study found stroke incidence to be associated with lower circulating IGF-I concentrations (43). Other studies have shown improved outcomes following ischemic stroke in participants with higher IGF-I concentrations (41). In hypertensive mice, liver-specific IGF-I-knockdown treatment was associated with increased and earlier incidence of cerebral microhemorrhages compared to control mice, likely due to impaired remodeling processes (44). Mechanisms underlying protective effects of IGF-I on the vasculature include anti-inflammatory properties, preservation of endothelial function, and a positive association with cardiovascular risk factors such as obesity and insulin resistance (43). While our findings did not reach conventional levels of statistical significance, effect sizes, consistency with previous research, and strong biological plausibility support further investigation.

Surprisingly, however, associations with the IGF-I/ IGFBP-3 ratio were opposite to those observed for IGF-I alone, with the ratio associated with reduced processing 


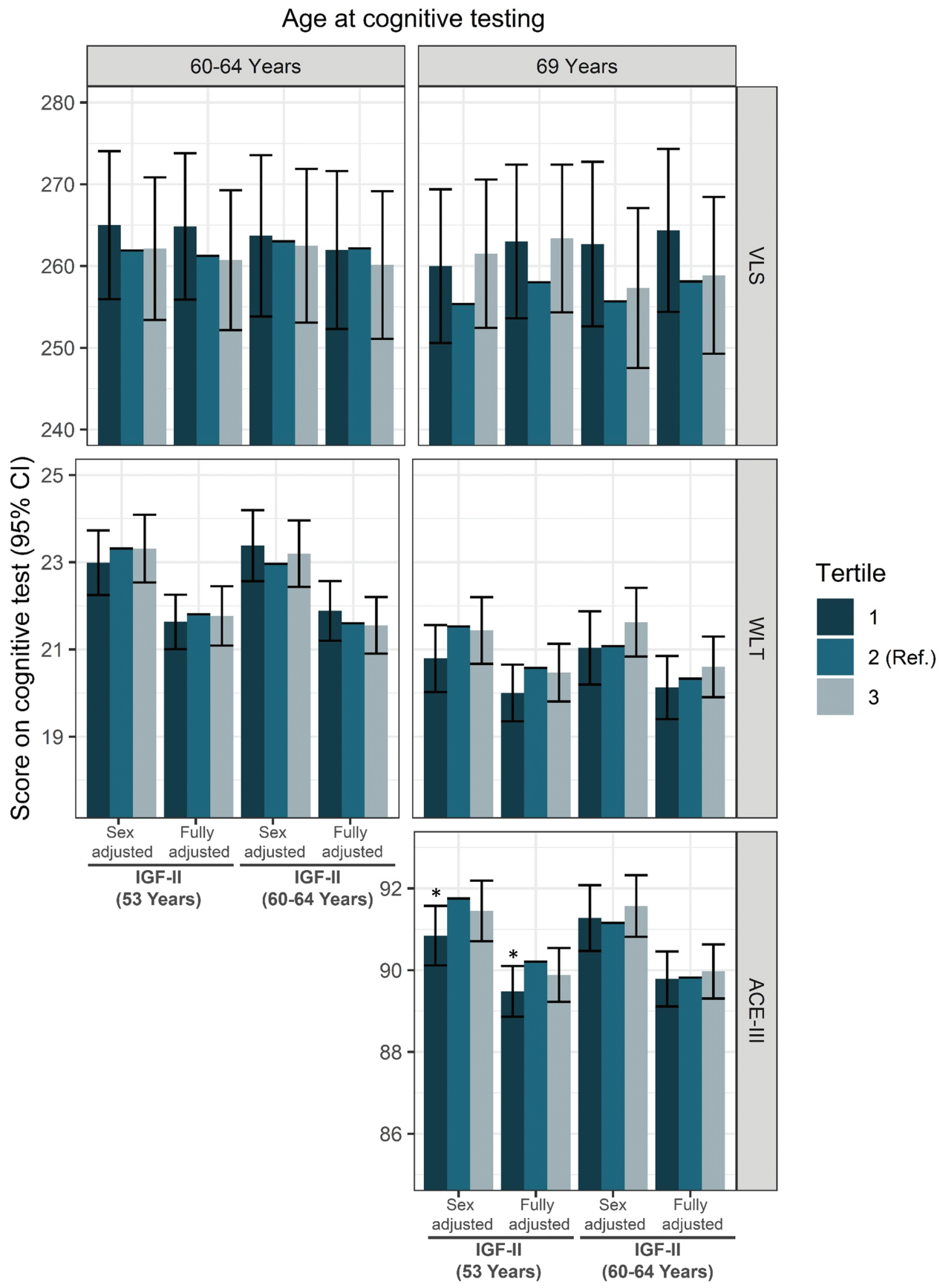

Figure 4. Predicted scores and 95\% confidence intervals for the effect of IGF-II tertiles at ages 53 years and 60 to 64 years on ACE-III, WLT, and VLS predicted score, in sex-adjusted and fully adjusted models (sex, early life cognition, lifetime education, smoking, and depressive symptoms). Tertile 2 presents the reference category used in the regression. ${ }^{*}$ significant at $P<0.05,{ }^{*}$ significant at $P<0.01$. Abbreviations: ACE-III, Addenbrookes Cognitive Exam III; IGF-I, insulin growth factor I; IGFBP-3, insulin growth factor binding protein 3; VLS, visual letter search test; WLT, word learning test.

speed and, to a lesser degree, cognitive state. This can be explained by IGFBP- 3 being positively associated with cognition independently of IGF-I. IGFBPs have been shown to execute IGF-I independent actions, including mediation of cell proliferation, apoptosis, and survival $(45,46)$. Furthermore, the age-related decline in IGFBP-3 concentration was markedly greater than that for IGF-I, resulting in an increase in IGF-I/IGFBP-3 ratio between the
2 timepoints. Thus, the apparent negative association with cognitive outcomes may be the product of the differential declines in the ratio components rather than a biologically relevant association of the ratio with the cognitive outcomes.

We observed globally improved cognitive outcomes associated with increased circulating IGFBP-3 concentrations. In line with our results, higher concentrations 
of IGFBP-3 were found to be cross-sectionally associated with better cognitive state in women in the Mayo Clinic Study of Ageing and reduced dementia incidence in men $(47,48)$. In mice, null mutations in the Igfbp 3 gene resulted in impaired neuronal structure and signaling and spatial working memory (49). However, findings are not consistent across studies with higher concentrations of IGFBP-3 related to poorer cognition in males in the Caerphilly Prospective Study (CaPS) (10). Our findings highlight the importance of exploring associations with individual factors that form a biologically meaningful molecular ratios and understanding age-related changes in exposures, which, as we show, may not be equivalent across associated measures.

In addition to favorable cognitive outcomes, IGFBP-3 was conjointly associated with a $10 \%$ reduction in WMHV in our study. Several studies have implied the possibility of a beneficial association between IGFBP-3 and cerebral small vessel disease. High concentrations of circulating IGFBP-3 are associated with reduced risk of ischemic stoke and improved functional outcomes following stroke events (50-52). In mice, IGFBP-3 was shown to protect retinal vasculature from hyperoxia-induced vessel regression and, in vitro, promoted differentiation of endothelial precursor cells to endothelial cells, indicating a potential pathway by which IGFBP-3 may reduce WMHV $(5,53)$.

Few studies have examined the relationship between IGF-II and cognitive outcomes. IGF-II has traditionally been seen as relatively unimportant beyond fetal development. However, there is increasing evidence relating detrimental IGF-II expression to disease after birth $(4,54)$. To our knowledge, only 1 other study has investigated the role of IGF-II on later-life cognition. The CaPS found a reduced occurrence of cognitive impairment without dementia with higher circulating IGF-II concentrations but not with cognitive state. In addition, CaPS did not find an association between IGF-I and cognitive function (10). In our study, we found a time-dependent association between higher IGF-II at age 53 and better visual memory and cognitive state at age 69 , presenting similarities to the results obtained from the IGF-I models. This suggests a common pathway, likely via signaling downstream of the IGF1R. Discrepancies between our findings and those from the CaPS may be driven by choice of outcome. The CaPS studied cognitive impairment-no dementia, dementia, and "normal" cognitive aging separately, therefore excluding participants with cognitive impairment-no dementia or dementia from models studying "normal" cognitive aging. It is possible that "normal" and "abnormal" cognitive aging are driven by separate mechanisms, and IGF-I and IGF-II might be protective in the context of "abnormal" cognitive aging only.
Our study has some important strengths, including a prospective design with extended follow-up (16 years) and important confounders, including childhood cognition. To our knowledge, it is the only study to use repeat exposure and outcome measures to better understand the temporal relationship between IGFs and cognition. Age-dependent relationships may reflect separate underlying biological pathways acting at different points in the life course. In addition, it is the first study to investigate both cognitive function and structural brain measures. However, it is important to consider some limitations of the present study. While associations were robust to adjustments, the observational methods used here cannot discount the possibility of residual confounding. For instance, this study did not adjust for chronic diseases that may impact IGF serum levels and associate with brain health independently of the IGF pathway (55).

To investigate active IGF-I, this study used the ratio of IGF-I to IGFBP-3. However, we acknowledge this to be an imprecise measure of IGF-I activity, as other binding proteins and pathway components are involved in regulating pathway activation. Furthermore, binding proteins have been shown to have IGF-I-independent actions (45). Conflicting results between IGF-I and IGF-I/IGFBP-3 in our study were seemingly explained by IGF-I-independent associations between IGFBP-3 and cognition, thereby supporting future study of IGFBP-3 independently from IGF-I. However, the use of "free" IGF-I may provide a more accurate view of the role of unbound circulating IGF-I on brain health (56).

Blood samples from ages 53 and 60 to 64 were assayed together and stored at $-80^{\circ} \mathrm{C}$ until quantification. Therefore, measures will have been stored for varying lengths of time, which may affect results, particularly if the protein is prone to degradation. However, IGF-I, IGF-II, and IGFBP-3 levels have previously been shown to remain stable following 9 years of $-80^{\circ} \mathrm{C}$ storage, which reduced the likelihood of this occurring in our study (57). Furthermore, biomarker concentrations may be affected by the differential fasting status when blood samples were collected at ages 53 (nonfasting) and 60 to 64 years (fasting). However, Bereket et al found no associations between IGF-I and IGFBP-3 concentration and short-term fasting, thus limiting the risk of fasting status being an important biasing factor in our study (58). Finally, with the latest follow-up at age 69, participants are still not old enough for severe cognitive decline to be common, which could mean limited statistical power to detect moderate to small associations.

In light of these findings, we would suggest further studies to determine the exact domains that may be affected by IGF-I, IGF-II, and IGFBP-3 concentrations. This could 
be done by using an array of cognitive tests that probe distinct cognitive domains. Further studies continuing to use repeat IGF and IGFBP exposures in relation to cognition measures across the life course may help provide a better understanding of the complex relationship between the 2. Re-examination of the relationships in the NSHD at an older age would be essential to understand age-dependent effects and potentially address the current inconsistencies in the field. Furthermore, genetic approaches to study the association between polygenic risk scores and cognition across the age spectrum may provide an alternate method to investigate relationships across the life course.

While repeat measures in our study have highlighted the importance of time of exposure, outcome, and follow-up period, further work is required to disentangle the temporal nature of the IIS-brain health relationship. Regular IGF-I, IGF-II, IGFBP-3, and cognition measures throughout the life course would help to elucidate this. As IGF-I concentrations vary with age, narrowing the age range at each sample collection could also prove essential $(59,60)$. The wide age range in a number of studies may prove responsible for some of the heterogeneity currently observed in results $(37,56,61)$.

In conclusion, in this UK birth cohort, higher circulating IGF-I and IGF-II levels were prospectively associated with a favorable cognitive state, while IGF-I/IGFBP-3 ratio was associated with slower processing speed. IGF-II was additionally associated with better memory performance. IGFBP-3 was independently associated with cognitive state, memory, processing speed, and WMHV. The present study has not only added to our understanding of the IIScognition relationship, but crucially, it has elucidated the importance of using a breadth of neuroimaging and cognitive function and state measures to understand more holistically how IGFs and IGFBPs relate to brain health from mid to later life.

\section{Acknowledgments}

The authors are grateful for the continued support of the NSHD study members without whom this study would not have been possible. We would also like to acknowledge Dr. Andy Wong for assistance in data extraction and management, and the Insight 46 study team.

Financial Support: This work was supported by the UK Medical Research Council, which provides core funding for the MRC Unit For Lifelong Health and Ageing at UCL and the MRC National Survey of Health and Development (MC_UU_00019/1) and supports AS, NC, DW, MR, and SNJ (MC_ST_LHA_2019; MC_UU_0019/2, MC_UU_0019/3). Insight 46 is principally funded by grants from Alzheimer's Research UK (and SNJ ARUK-PG2014-1946, ARUKPG2017-1946), the Medical Research Council Dementias Platform UK (CSUB19166), the Wolfson Foundation (PR/ylr/18575) and the
Drake Foundation. JMS acknowledges the EPSRC (EP/J020990/1) and European Union's Horizon 2020 research and innovation program (Grant 666992) and a Weston Brain Institute and Selfridges Group Foundation award (176724). DC is funded by the National Institute on Aging (RO1AG017644) and ESRC (ES/S013830/1). CHS is supported by an Alzheimer's Society Junior Fellowship (ASJF-17-011). VG is funded by Joint Diabetes UK and British Heart Foundation (15/0005250).

\section{Additional Information}

Correspondence: Antoine Salzmann, MRC Unit for Lifelong Health and Ageing at UCL, University College London, 5th floor, 1-19 Torrington Place, London, WC1E 7HB, London, UK. Email: antoine.salzmann.14@ucl.ac.uk.

Disclosures: JMS has received research funding from Avid Radiopharmaceuticals (a wholly owned subsidiary of Eli Lilly); consulted for Roche Pharmaceuticals, Biogen, and Eli Lilly; and given educational lectures sponsored by GE, Eli Lilly, and Biogen and serves on a Data Safety Monitoring Committee for Axon Neuroscience SE. NC receives funds for serving on a Data Safety Monitoring Committee for a study sponsored by AstraZeneca. All other authors have no conflicts of interest to declare.

Data Availability: Restrictions apply to the availability of some or all data generated or analyzed during this study to preserve patient confidentiality or because they were used under license. The corresponding author will, on request, detail the restrictions and any conditions under which access to some data may be provided.

\section{References}

1. Milman S, Huffman DM, Barzilai N. The somatotropic axis in human aging: framework for the current state of knowledge and future research. Cell Metab. 2016;23(6):980-989.

2. Baxter RC. Insulin-like growth factor (IGF)-binding proteins: interactions with IGFs and intrinsic bioactivities. Am J Physiol Endocrinol Metab. 2000;278(6):E967-E976.

3. Juul A, Main K, Blum WF, Lindholm J, Ranke MB, Skakkebaek NE. The ratio between serum levels of insulin-like growth factor (IGF)-I and the IGF binding proteins (IGFBP-1, 2 and 3 ) decreases with age in healthy adults and is increased in acromegalic patients. Clin Endocrinol (Oxf). 1994;41(1):85-93.

4. Chao W, D'Amore PA. IGF2: epigenetic regulation and role in development and disease. Cytokine Growth Factor Rev. 2008;19(2):111-120.

5. Jogie-Brahim S, Feldman D, Oh Y. Unraveling insulin-like growth factor binding protein-3 actions in human disease. Endocr Rev. 2009;30(5):417-437.

6. Dyer AH, Vahdatpour C, Sanfeliu A, Tropea D. The role of insulin-like growth factor 1 (IGF-1) in brain development, maturation and neuroplasticity. Neuroscience. 2016;325:89-99.

7. Abuzzahab MJ, Schneider A, Goddard A, et al; Intrauterine Growth Retardation (IUGR) Study Group. IGF-I receptor mutations resulting in intrauterine and postnatal growth retardation. N Engl J Med. 2003;349(23):2211-2222.

8. Frater J, Lie D, Bartlett P, McGrath JJ. Insulin-like growth factor 1 (IGF-1) as a marker of cognitive decline in normal ageing: a review. Ageing Res Rev. 2018;42:14-27. 
9. Gubbi S, Quipildor GF, Barzilai N, Huffman DM, Milman S. 40 years of IGF1: IGF1: the Jekyll and Hyde of the aging brain. J Mol Endocrinol. 2018;61(1):T171-T185.

10. Green CJ, Holly JM, Bayer A, et al. The role of IGF-I, IGF-II, and IGFBP-3 in male cognitive aging and dementia risk: the Caerphilly Prospective Study. J Alzheimers Dis. 2014;41(3):867-875.

11. Kuh D, Wong A, Shah I, et al. The MRC National Survey of Health and Development reaches age 70: maintaining participation at older ages in a birth cohort study. Eur J Epidemiol. 2016;31(11):1135-1147.

12. Wadsworth M, Kuh D, Richards M, Hardy R. Cohort profile: the 1946 National Birth Cohort (MRC National Survey of Health and Development). Int J Epidemiol. 2006;35(1):49-54.

13. Lane CA, Parker TD, Cash DM, et al. Study protocol: Insight 46: a neuroscience sub-study of the MRC National Survey of Health and Development. BMC Neurol. 2017;17(1):75.

14. Leung KK, Barnes J, Modat M, et al; Alzheimer's Disease Neuroimaging Initiative. Brain MAPS: an automated, accurate and robust brain extraction technique using a template library. Neuroimage. 2011;55(3):1091-1108.

15. Jorge Cardoso M, Leung K, Modat M, et al; Alzheimer's Disease Neuroimaging Initiative. STEPS: Similarity and Truth Estimation for Propagated Segmentations and its application to hippocampal segmentation and brain parcelation. Med Image Anal. 2013;17(6):671-684.

16. Sudre CH, Cardoso MJ, Bouvy WH, Biessels GJ, Barnes J, Ourselin S. Bayesian model selection for pathological neuroimaging data applied to white matter lesion segmentation. IEEE Trans Med Imaging. 2015;34(10):2079-2102.

17. Lane CA, Barnes J, Nicholas JM, et al. Associations between vascular risk across adulthood and brain pathology in late life: evidence from a British birth cohort. JAMA Neurol. 2020;77(2):175-183.

18. Lane CA, Barnes J, Nicholas JM, et al. Associations between blood pressure across adulthood and late-life brain structure and pathology in the neuroscience substudy of the 1946 British Birth Cohort (Insight 46): an epidemiological study. Lancet Neurol. 2019;18(10):942-952.

19. Lemmey A, Maddison P, Breslin A, et al. Association between insulin-like growth factor status and physical activity levels in rheumatoid arthritis. J Rheumatol. 2001;28(1):29-34.

20. Bann D, Holly JMP, Lashen H, et al. Changes in insulin-like growth factor-I and -II associated with fat but not lean mass in early old age. Obes Silver Spring Md. 2015;23(3):692-698.

21. Kucera R, Topolcan O, Pecen L, et al. Reference values of IGF1, IGFBP3 and IGF1/IGFBP3 ratio in adult population in the Czech Republic. Clin Chim Acta. 2015;444:271-277.

22. Richards M, Barnett JH, Xu MK, et al; MRC National Survey of Health and Development Scientific and Data Collection Team. Lifetime affect and midlife cognitive function: prospective birth cohort study. Br J Psychiatry. 2014;204(3):194-199.

23. Richards M, Shipley B, Fuhrer R, Wadsworth ME. Cognitive ability in childhood and cognitive decline in mid-life: longitudinal birth cohort study. BMJj. 2004;328(7439):552.

24. Hsieh S, Schubert S, Hoon C, Mioshi E, Hodges JR. Validation of the Addenbrooke's Cognitive Examination III in frontotemporal dementia and Alzheimer's disease. Dement Geriatr Cogn Disord. 2013;36(3-4):242-250.
25. Kuh D, Hardy R, Richards M, et al. MRC NSHD 2013-2018 data. Published online 2016. doi:10.5522/NSHD/Q103

26. Richards M, James SN, Sizer A, et al. Identifying the lifetime cognitive and socioeconomic antecedents of cognitive state: seven decades of follow-up in a British birth cohort study. BMJ Open. 2019;9(4):e024404.

27. Kaklamani VG, Linos A, Kaklamani E, Markaki I, Mantzoros C. Age, sex, and smoking are predictors of circulating insulin-like growth factor 1 and insulin-like growth factor-binding protein 3. J Clin Oncol. 1999;17(3):813-813.

28. Chigogora S, Zaninotto P, Kivimaki M, Steptoe A, Batty GD. Insulinlike growth factor 1 and risk of depression in older people: the English Longitudinal Study of Ageing. Transl Psychiatry. 2016;6(9):e898.

29. Hardy R, Maddock J, Ghosh AK, Hughes AD, Kuh D. The relationship between pubertal timing and markers of vascular and cardiac structure and function in men and women aged 60-64 years. Sci Rep. 2019;9:11037.

30. Goldberg DP, Hillier VF. A scaled version of the General Health Questionnaire. Psychol Med. 1979;9(1):139-145.

31. James SN, Davis D, O'Hare C, et al. Lifetime affective problems and later-life cognitive state: over 50 years of follow-up in a British birth cohort study. J Affect Disord. 2018;241:348-355.

32. van Buuren S, Groothuis-Oudshoorn K. Mice: multivariate imputation by chained equations in R. J Stat Softw. 2011;45(1):1-67.

33. R Core Team. R: A Language and Environment for Statistical Computing. Vienna, Austria: R Foundation for Statistical Computing; 2018. https://www.R-project.org/

34. Davis D, Bendayan R, Muniz Terrera G, Hardy R, Richards M, Kuh D. Decline in search speed and verbal memory over 26 years of midlife in a British Birth Cohort. Neuroepidemiology. 2017;49(3-4):121-128.

35. Okereke O, Kang JH, Ma J, Hankinson SE, Pollak MN, Grodstein F. Plasma IGF-I levels and cognitive performance in older women. Neurobiol Aging. 2007;28(1):135-142.

36. Perice L, Barzilai N, Verghese J, et al. Lower circulating insulinlike growth factor-I is associated with better cognition in females with exceptional longevity without compromise to muscle mass and function. Aging (Albany, NY). 2016;8(10):2414-2424.

37. Tumati S, Burger H, Martens S, van der Schouw YT, Aleman A. Association between cognition and serum insulin-like growth factor-1 in middle-aged \& older men: an 8 year follow-up study. PLoS One. 2016;11(4):e0154450.

38. Piriz J, Muller A, Trejo JL, Torres-Aleman I. IGF-I and the aging mammalian brain. Exp Gerontol. 2011;46(2-3):96-99.

39. Serhan A, Aerts JL, Boddeke EWGM, Kooijman R. Neuroprotection by insulin-like growth factor- 1 in rats with ischemic stroke is associated with microglial changes and a reduction in neuroinflammation. Neuroscience. 2020;426:101-114.

40. Deng W, Aimone JB, Gage FH. New neurons and new memories: how does adult hippocampal neurogenesis affect learning and memory? Nat Rev Neurosci. 2010;11(5):339-350.

41. Fernandez AM, Torres-Alemán I. The many faces of insulinlike peptide signalling in the brain. Nat Rev Neurosci. 2012;13(4):225-239.

42. Westwood AJ, Beiser A, Decarli C, et al. Insulin-like growth factor-1 and risk of Alzheimer dementia and brain atrophy. Neurology. 2014;82(18):1613-1619. 
43. Saber H, Himali JJ, Beiser AS, et al. Serum insulin-like growth factor 1 and the risk of ischemic stroke: the Framingham study. Stroke. 2017;48(7):1760-1765.

44. Tarantini S, Valcarcel-Ares NM, Yabluchanskiy A, et al. Insulinlike growth factor 1 deficiency exacerbates hypertension-induced cerebral microhemorrhages in mice, mimicking the aging phenotype. Aging Cell. 2017;16(3):469-479.

45. Allard JB, Duan C. IGF-binding proteins: why do they exist and why are there so many? Front Endocrinol. 2018;9:117.

46. Baxter RC. Insulin-like growth factor binding protein-3 (IGFBP3): novel ligands mediate unexpected functions. J Cell Commun Signal. 2013;7(3):179-189.

47. Wennberg AMV, Hagen CE, Machulda MM, et al. The association between peripheral total IGF-1, IGFBP-3, and IGF-1/ IGFBP-3 and functional and cognitive outcomes in the Mayo Clinic Study of Aging. Neurobiol Aging. 2018;66:68-74.

48. Almeida OP, Hankey GJ, Yeap BB, Paul Chubb SA, Gollege J, Flicker L. Risk of prevalent and incident dementia associated with insulin-like growth factor and insulin-like growth factorbinding protein 3. Mol Psychiatry. 2018;23(8):1825-1829.

49. Dai H, Goto YI, Itoh M. Insulin-like growth factor binding protein-3 deficiency leads to behavior impairment with monoaminergic and synaptic dysfunction. Am J Pathol. 2017;187(2):390-400.

50. Åberg D, Jood K, Blomstrand C, et al. Serum IGF-I levels correlate to improvement of functional outcome after ischemic stroke. J Clin Endocrinol Metab. 2011;96(7):E1055-E1064.

51. Johnsen SP, Hundborg HH, Sørensen HT, et al. Insulin-like growth factor (IGF) I, -II, and IGF binding protein-3 and risk of ischemic stroke. J Clin Endocrinol Metab. 2005;90(11):5937-5941.

52. Armbrust M, Worthmann H, Dengler R, et al. Circulating insulin-like growth factor-1 and insulin-like growth factor binding protein-3 predict three-months outcome after ischemic stroke. Exp Clin Endocrinol Diabetes. 2017;125(7):485-491.
53. Chang KH, Chan-Ling T, McFarland EL, et al. IGF binding protein-3 regulates hematopoietic stem cell and endothelial precursor cell function during vascular development. Proc Natl Acad Sci U S A. 2007;104(25):10595-10600.

54. Reik W, Constancia M, Dean W, et al. Igf2 imprinting in development and disease. Int J Dev Biol. 2000;44(1):145-150.

55. Min DY, Jung E, Kim J, Lee YH, Shin SY. Leptin stimulates IGF-1 transcription by activating AP-1 in human breast cancer cells. BMB Rep. 2019;52(6):385-390.

56. Kalmijn S, Janssen JA, Pols HA, Lamberts SW, Breteler MM. A prospective study on circulating insulin-like growth factor I (IGF-I), IGF-binding proteins, and cognitive function in the elderly. J Clin Endocrinol Metab. 2000;85(12):4551-4555.

57. Ito Y, Nakachi K, Imai K, et al. Stability of frozen serum levels of insulin-like growth factor-I, insulin-like growth factor-II, insulin-like growth factor binding protein-3, transforming growth factor $\beta$, soluble fas, and superoxide dismutase activity for the JACC study. J Epidemiol. 2005;15(Supplement_I):S67 -S73.

58. Bereket A, Wilson TA, Blethen SL, et al. Effect of short-term fasting on free/dissociable insulin-like growth factor I concentrations in normal human serum. J Clin Endocrinol Metab. 1996;81(12):4379-4384.

59. Yamamoto H, Sohmiya M, Oka N, Kato Y. Effects of aging and sex on plasma insulin-like growth factor I (IGFI) levels in normal adults. Acta Endocrinol (Copenh). 1991;124(5):497-500.

60. Joseph D'Ercole A, Ye P. Expanding the mind: insulin-like growth factor I and brain development. Endocrinology. 2008;149(12):5958-5962.

61. Al-Delaimy WK, von Muhlen D, Barrett-Connor E. Insulinlike growth factor-1, insulinlike growth factor binding protein-1, and cognitive function in older men and women. J Am Geriatr Soc. 2009;57(8):1441-1446. 